\title{
The Lewisham and Greenwich idiopathic intracranial hypertension cohort: a retrospective study
}

\author{
Authors: Osman Younus, ${ }^{\mathrm{A}}$ Bazo Raheem, ${ }^{\mathrm{A}}$ Graziella Quattrocchi, ${ }^{\mathrm{B}}$ Timothy Hampton, ${ }^{\mathrm{A}}$ Georgios Dervenoulas ${ }^{\mathrm{A}}$ and \\ Eli Silber ${ }^{A}$
}

\section{Introduction}

Idiopathic intracranial hypertension (IIH) is a syndrome of raised intracranial pressure without hydrocephalus or mass lesion and normal cerebrospinal fluid (CSF) constituents. Incidence and prevalence of $\mathrm{IIH}$ are rising, and this is anticipated to have a significant economic impact on healthcare systems. ${ }^{1}$ We reviewed the Lewisham and Greenwich NHS Trust experience of IIH over the past 6 years. We sought to characterise a cohort of patients and explore the admissions rate, assess the influence of medical and psychiatric comorbidities and determine the health economic impact of IIH.

\section{Methods}

We systematically searched the hospital database for new diagnoses of IIH from 2013-19 across hospital sites at the Lewisham and Greenwich NHS Trust. IIH was defined using the modified Dandy criteria. ${ }^{2}$ Data were collected retrospectively and included patient demographics, body mass index, number of admissions for IIH, visual assessment at diagnosis and most recent follow-up, treatment, and medical and psychiatric comorbidity. Cost analysis was based upon NHS tariff values for the time of admission.

\section{Results and discussion}

A total of 47 new diagnoses of IIH were identified from 2013-19. The average age of patients was 29 years and $96 \%$ were female (45/47). The number of new cases in 2018 was 3.5 times higher than in 2013.

There were 120 admissions for 36 patients. Eleven patients were managed as outpatients. Nineteen patients (40\%) were admitted on more than one occasion, consistent with a national rate of $38 \% .^{3}$ The annual number of admissions rose by a factor of 22 from 2013 to 2019 ( 1 to 22). The annual relapse rate was 1.75. The mean length of stay was 8.61 days, four times greater than the national average of $2 . .^{3}$

Authors: ${ }^{A}$ Queen Elizabeth Hospital, Woolwich, UK; ${ }^{\mathrm{B}}$ University Hospital Lewisham, London, UK
The most common medical comorbidities were gynaecological $(21 / 47 ; 45 \%)$ and respiratory $(12 / 47 ; 25 \%)$ conditions. A psychiatric history was present in $38 \%(18 / 47)$ of patients.

More than half of the patients required therapeutic lumbar puncture in addition to medical therapy (55\%). The average number of therapeutic lumbar punctures was 2.62. Two patients (4\%) were referred for CSF diversion procedures. Only 8.5\% (4/47) of patients reported an intolerance or non-compliance to medication. Thirteen patients $(28 \%)$ had a history of medication use associated with causing IIH.

The mean body mass index was $37 \mathrm{~kg} / \mathrm{m}^{2}$. Weight loss was reported by $17 \%(8 / 47)$ of patients. Only $15 \%$ (7/47) of patients were referred to a dietitian.

Ophthalmology assessment was available for 39 patients. Of these, $77 \%$ (30/39) had papilloedema on initial presentation. This resolved on the most recent ocular assessment in $43 \%$ $(13 / 30)$. The average initial visual acuity was measured at 6/7.5. The most recent average visual acuity was $6 / 6$.

The annual hospital costs of IIH rose 50 times over a 6-year period, from $\mathfrak{E} 1,316.78$ to $\mathfrak{E} 66,879.14$.

\section{Conclusion}

The number of new cases of IIH is rising. Admission rates are rising yearly. This is unlikely to improve with the global obesity epidemic. These findings highlight the unmet need for an efficient pathway to reduce admissions and focus on prevention.

\section{Conflicts of interest}

None declared.

\section{References}

1 Mollan SP, Ali F, Hassan-Smith G et al. Evolving evidence in adult idiopathic intracranial hypertension: pathophysiology and management. J Neurol Neurosurg Psychiatry 2016;87:982-92.

2 Friedman DI, Liu GT, Digre KB. Revised diagnostic criteria for the pseudotumor cerebri syndrome in adults and children. Neurology 2013;81:1159-65.

3 Mollan SP, Aguiar M, Evison F, Frew E, Sinclair AJ. The expanding burden of idiopathic intracranial hypertension. Eye 2019;33:478-85. 